\title{
Pathological effects of staphylococcal species ( $S$. aureus, S. xylosus and S. lentus) on some visceral organs (liver, kidney and bladder) of wistar rats following intraperitoneal inoculation
}

\author{
Onyebueke Ebere Adaeze ${ }^{1 \star}$, Onyemelukwe Ngozi Felicia ${ }^{1}$, Achukwu Peter Uwadiegwu ${ }^{1}$ and \\ Onuba Augustine Chukwudum ${ }^{2}$ \\ ${ }^{1}$ Department of Medical Laboratory Sciences, Faculty of Health Sciences and Technology, College of Medicine, \\ University of Nigeria, Enugu Campus, Enugu State, Nigeria. \\ ${ }^{2}$ Department of Veterinary Surgery and Radiology, Faculty of Veterinary Medicine, University of Nigeria Nsukka, Enugu \\ State, Nigeria.
}

Received 16 August, 2017; Accepted 12 January, 2018

\begin{abstract}
Following the intraperitoneal inoculation of 200 Wistar rats (96 males, 96 females and 8 controls) aged 2 to 4 months with graded-doses of saline and peptone water broth cultures of Staphylococcus aureus, Staphylococcus xylosus and Staphylococcus lentus isolates obtained from urinary tract infection (UTI) in Enugu, Nigeria and typed down to species using API@ Staph typing kit, the rats were observed for 72 $h$ and were euthanized. The liver, kidney and bladder were harvested and processed histologically. Out of the 96 rats (48 males and 48 females) inoculated with peptone water broth cultures of $S$. aureus 834 , S. xylosus 837, S. aureus 856 and S. lentus 853 strains, $12(12.5 \%)$ died, with $8(66.7 \%)$ from S. xylosus 837 and $4(33.3 \%)$ from $S$. aureus 856 but no death from S. aureus 834 and $S$. lentus 853 . More males $6(75 \%)$ died from S. xylosus 837 than females $2(25 \%)$ while more females $4(100 \%)$ died from $S$. aureus 856 than males $0(0 \%)$. No death occurred from inoculation of another 96 rats with saline broth culture of the test strains. Evidence of necrosis of the liver parenchyma with infiltration of the inflammatory cells around the pericentral areas upon S. aureus inoculation was observed. S. xylosus and S. lentus showed no histological damage to the liver. In the kidney, $S$. aureus produced tubular casts, erosions and glomerular oedema. S. xylosus and S. lentus produced tubular casts, glomerular distortions and oedema. The bladder showed mild effect on the musculature with $S$. aureus and none for $S$. xylosus and S. lentus, respectively.
\end{abstract}

Key words: Staphylococcus species, liver, kidney, bladder, intraperitoneal inoculation, mortality rate and damage.

\section{INTRODUCTION}

Staphylococci are Gram-positive spherical cells, usually arranged in grapelike irregular clusters. The genus Staphylococcus has at least 40 species (Brooks et al., 2013) amongst which are Staphylococcus aureus, Staphylococcus xylosus and Staphylococcus lentus. Most are harmless and reside normally on the skin and mucous membranes of humans and other animals, found worldwide, they are small components of soil microbial flora (Madigan and Martinko, 2005).

Humans are the reservoir for staphylococci. The nose is the main site of colonization of Staphylococcus aureus 
and approximately $30 \%$ of people are colonized at any one time. The skin especially of hospital personnel and patients is also a common site of $S$. aureus colonization. $S$. aureus is also found in the vagina of approximately $5 \%$ of women (Levinson, 2012). However, S. aureus is a cause of many pyogenic (pus producing) and toxin mediated diseases and a major cause of skin, soft tissue, bone, joint, lung, heart and kidney infections (Levinson, 2012). S. aureus have also been implicated in several literatures as a cause of urinary tract infection (UTI) (Oladeinde et al., 2011; Alemu et al., 2012; Amaeze et al., 2013; Pragash et al., 2014).

$S$. aureus, a coagulase positive staphylococcus is able to cause diseases due to possession of several virulence factors such as extacellular enzymes and toxins produced by strains of the organism which contributes to its invasiveness and pathogencity and this includes coagulase, haemolysins, leucocidin, staphylokinases, lipase, hyaluronidase, protein $A$, enterotoxins, and toxic shock syndrome toxin (Cheesbrough, 2010).

Although coagulase negative staphylococci (CoNS) are normal human microbiota, they sometimes cause infection often associated with implanted devices such as joint prosthesis, shunts and intravascular catheters especially in very young, old and immuno compromised patients (Brooks et al., 2013).

S. xylosus, a coagulase negative Staphylococcus has been associated with nasal dermatitis in gerbrils, pyelonephritis in humans, avian Staphylococcosis, bovine intermammary infection (Wikipedia the free encyclopaedia, 2013), and urinary tract infection (Bolaji et al., 2013). S. xylosus is able to cause disease due to possession of virulence factors such as haemolysin, protease, urease (Al-Mathkhury et al., 2008), and lipase (Mosbah et al., 2006). S. xylosus isolates from UTI have been reported to cause several pathological changes in mice (Al-Mathkhury et al., 2008). S. lentus, a coagulase negative staphylococcus has been isolated from urine (Bolaji et al., 2013) but not much is known concerning its pathogenicity.

$S$. aureus is the most reported of the staphylococcal species in UTI in clinical laboratories and in research works in the present study area and elsewhere while other CoNS encountered are usually disregarded as not able to cause UTI, are unreported and when reported they are most often not typed to species, lumped together as CoNS with very few cases where they are typed. The increasing use of medical devices such as shunts and heart valves can predispose to septicemia with any of the staphylococcal species (Levinson, 2012) and can lead to pyelonephritis while the use of urinary catheters predisposes to staphylococcal UTI.

There is paucity of documented works on the pathological effects of $S$. aureus, $S$. xylosus and S. lentus isolates from UTI on the visceral organs of experimental animals in the present study area which could help one to infer the possible effects of these staphylococcal species on the visceral organs of humans especially liver, kidney and bladder. This will help to substantiate the claims that these staphylococci cause UTI and show the need for our clinical laboratories to type them to species level and report on them in UTI cases.

This study therefore is aimed at investigating the pathological effects of $S$. aureus, $S$. xylosus and $S$. lentus isolates from UTI on the visceral organs (Liver, Kidney and bladder) of Wistar rats following intraperitoneal inoculation to determine which of these organs may be affected by them and the extent of damage that may be encountered.

\section{MATERIALS AND METHODS}

\section{Isolation of staphylococcal species}

After obtaining ethical clearance from the ethics committees of the University of Nigeria Teaching Hospital (UNTH) Ituku-Ozalla and Enugu State University Teaching Hospital (ESUTH) Enugu, both in Enugu State, South East Nigeria and informed consents from the participants or their wards, 818 participants were investigated for staphylococcal UTI. Their urine samples were cultured onto blood agar and MacConkey agar plates, and the plates were incubated for $24 \mathrm{~h}$ at $37^{\circ} \mathrm{C}$. At the end of the incubation period, the plates were examined macroscopically and samples showing uniform growth $\geq 10^{5} \mathrm{CFU} / \mathrm{ml}$ of urine were regarded as significant for UTI and subjected to further tests including gram reaction and standard biochemical tests. Isolates suspected to be Staphylococcus species (spp) were further tested using DNase test with deoxyribonuclease test agar (Fluka BioChemika), novobiocin (Oxoid) sensitivity test and 'Staph speciation test' using the $\mathrm{API}^{\circledR}$ Staph test kit (Biomerieux France) while the identity of the staphylococcal spp were confirmed using the apiweb ${ }^{\mathrm{TM}}$ identification software (Biomerieux France) (Ochei and Kolhatkar, 2000; Bolaji et al., 2013). Out of the samples examined, 89 were positive for staphylococcal UTI. From the most commonly incriminated staphylococcal spp. in the UTI cases ( $S$. aureus, $S$. xylosus and $S$. lentus), two strains of $S$. aureus and one strain each of $S$. xylosus and $S$. lentus, were used for the pathological study.

\section{Study design}

The study adopted an experimental design by intraperitoneal injection of the 4 test strains of Staphylococus (two strains of $S$. aureus and one strain each of $S$. xylosus and $S$. lentus) into albino Wistar rats. Methods for intraperitoneal injection were according to Janakat et al. (2002).

\section{Animal procurement and housing}

Two hundred laboratory reared Wistar rats (100 males and 100 females) obtained from the animal unit of the University of Nigeria,

*Corresponding author. E-mail: ebere.onyebueke@unn.edu.ng. Tel: +2348033500885.

Author(s) agree that this article remains permanently open access under the terms of the Creative Commons Attribution License 4.0 International License 
Enugu campus were housed in the animal houses of the unit and were allowed to acclimatize for 14 days while feeding them with grower feed before inoculation.

\section{Preparation of inoculums}

Four strains of three staphylococcal species isolated from UTI in this study $(S$. aureus, 834, S. xylosus 837, S. aureus 856 and $S$. lentus 853) were used for the study. The strains of the Staphylococcal species used were previously confirmed in the Microbiology Laboratory of the Department of Medical Laboratory Sciences of the University of Nigeria Enugu Campus using standard microbiology biochemical tests, Novobicin ${ }^{\circledR}$ drug sensitivity test, API ${ }^{\circledR}$ Staph test Kit (Biomerieux France) ref no 20500 and the apiweb $^{\mathrm{TM}}$ identification software (Biomerieux France) both for the identification of staphylococci, micrococcus and related genera. The isolates were maintained in nutrient agar slants in MacCartney bottles at 2 to $4^{\circ} \mathrm{C}$ in the Microbiology laboratory of the Department of Medical Laboratory Sciences of the University of Nigeria Enugu Campus. Prior to use, the strains were subculture onto fresh nutrient agar slants which were incubated aerobically for $24 \mathrm{~h}$ at $37^{\circ} \mathrm{C}$. The colonies of each of the four strains of the Staphylococcus species were homogenized in sterile peptone water broth and sterile saline water broth respectively, and their turbidities adjusted to correspond to 0.5 MacFarlands turbidity standard (Equivalent to $1 \times 10^{8} \mathrm{CFU} / \mathrm{ml}$ ).

\section{Animal grouping and inoculation}

The 200 Wistar rats (100 males and 100 females) were placed in 18 cages with the first 16 cages ( 1 to 16) containing 12 Wistar rats each while the last 2 cages (17 and 18) which served as controls contained 4 Wistar rats each. Virulence was assessed using graded doses of the Staphylococcus species $(0.2,0.5,1.0,0.0$ control) $\mathrm{ml}$ $/ \mathrm{kg}$ body weight of the rats in the experiment. Intraperitoneal inoculation (ip) of both the saline broth as well as the peptone water broth of the staphylococcal test species were conducted on the rats. The rats were weighed before inoculation. Cages 1 to 4 (Female rats) and cages 5 to 8 (male rats) contained 12 rats each (total $=96$ rats). Rats in cages $1,2,3$ and 4 (female rats) were inoculated intraperitoneally with peptone water broth culture of $S$. aureus 834, S. xylosus 837, S. aureus 856 and S. lentus 853 respectively. In each of the 4 cages, the rats were grouped into three groups of 4 rats each $(A, B, C)$, and the 3 groups were inoculated with graded doses of the strains of the test staphylococcal species $(A=0.2 \mathrm{ml}, B=0.5 \mathrm{ml} C=1.0 \mathrm{ml})$ respectively. Same inoculation procedure was repeated for rats in cages 5, 6, 7 and 8 (male rats). The same type of grouping and inoculation procedure was performed for another set of rats in cages 9 to 12 (female rats) and cages 13 to 16 (male rats) which were inoculated intraperitoneally with graded doses of saline broth culture of the 4 strains of the test staphylococcal species. Cages 17 (female rats) and 18 (male rats) containing 4 rats each served as control and were not inoculated with any both culture.

\section{General observation and sample collection}

The animals were observed for $72 \mathrm{~h}$ (3days) post infection (dpi) while feeding them with their food and water. Subsequently, they were individually weighed and sacrificed. The visceral organs of the rats (liver, kidney and bladder) were harvested, preserved in formalin and sent quickly to the histology laboratory for tissue processing.

\section{Histopathology}

The formalin fixed visceral organs of the rats (liver, kidney and bladder) were processed histologically by paraffin wax embedding technique, stained with Erlirch's Haematoxylin and eosin stain, and mounted in DPX. The processed tissues were histologically examined.

\section{RESULTS}

The result of the present study showed that out the 96 rats (48 males and 48 females) inoculated intraperitoneally with graded doses $(0.2,0.5,1.0)$ of the staphylococcal species in peptone water broth ( $S$. aureus 834, S. xylosus 837, S.aureus 856 and S. lentus 853), 12 died showing a mortality rate of $12.5 \%$. No death was recorded from intraperitoneal inoculation of the other 96 rats with saline broth of the four strains of Staphylococcus species tested. Of the 12 deaths encountered in peptone water broth inoculation of the staphylococcal strains, $8(66.7 \%)$ came from $S$. xylosus 837 inoculation while 4 (33.3\%) was from S. aureus 856 inoculation but no death was recorded from $S$. aureus 834 and S. lentus 853 peptone water broth inoculation.

Out of the 24 Wistar rats (12 males and 12 females) inoculated with graded doses of S. xylosus 837, 8 died with a mortality rate of $33.3 \%$. Of this 8 , males incurred more deaths $(6(75 \%))$ than females $(2(25 \%))$. Female verses male mortality ratio was 1:3. 24 Wistar rats were also inoculated with graded doses of $S$. aureus (856) of which four deaths occurred with a mortality rate of $14.7 \%$. Deaths occurring only in females $(4(100 \%))$ and non in males $(0 \%)$ showing a female versus male ratio of $4: 0$.

For $S$. xylosus 837 , of the eight deaths that occurred, none (0) occurred at a dose of $0.2 \mathrm{ml}$ of the inoculum, 4 deaths occurred at $0.5 \mathrm{ml}$ ( 2 females and 2 males) while 4 deaths occurred at $1.0 \mathrm{ml}$ inoculum (males only). For $S$. aureus 856, out of the 4 deaths that occurred, none occurred at $0.2 \mathrm{ml}$ and at $0.5 \mathrm{ml}$ doses of the inoculum, but the four deaths occurred at $1.0 \mathrm{ml}$ of the inoculum. This study was designed to assess the pathogenesis and virulence of $S$. aureus, $S$. xylosus, and $S$. lentus in the kidney, bladder (urinary system) and the liver following intraperitoneal inoculation of Wistar rats which produces some tissue alterations. The virulence was assessed using graded doses of the staphylococcal strains $(0.2$, $0.5,1.0$ and 0.0 control) $\mathrm{ml} / \mathrm{kg}$ body weight of the rats in experiments $A, B, C$ and $D$ respectively, after $72 \mathrm{~h}$. D served as control and as such was not inoculated.

In group 1 model of the experiment $A B C$ using graded doses of $S$. aureus on the Wistar rats, the histology of the liver parenchyma remained intact (Plate 1). There was occasional mild infiltration of the inflammatory cells (Plate 2) around the peri-central areas of the liver. The kidney demonstrated a higher degree of toxigenic modification. There was presence of tubular casts in varying degree, tubular erosion with inflammatory cells (Plate 3 ) and 


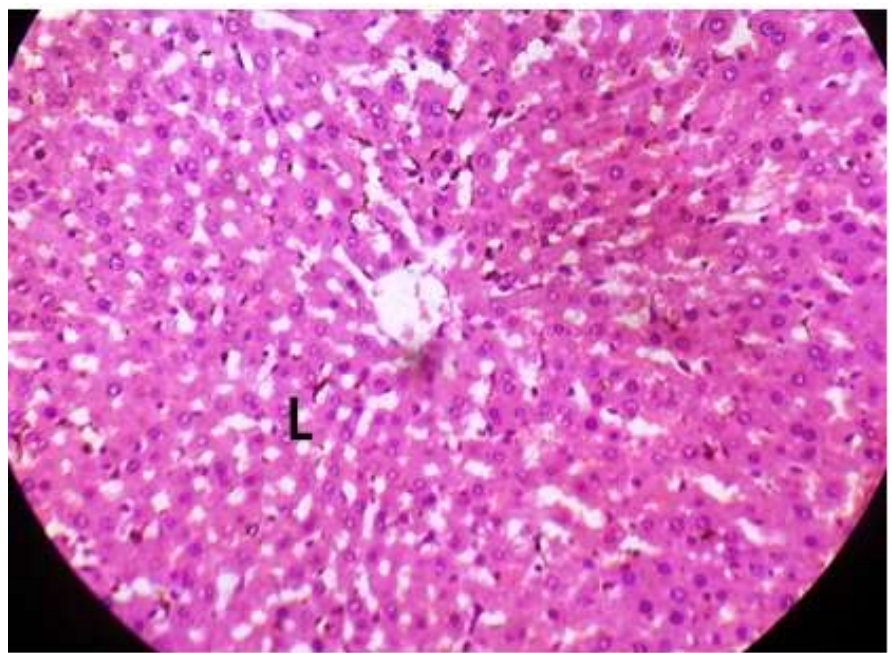

Plate 1. A piece of liver tissue of rat showing intact liver parenchyma $(\mathrm{L})$ after inoculation with $S$. aureus. H/E x200.

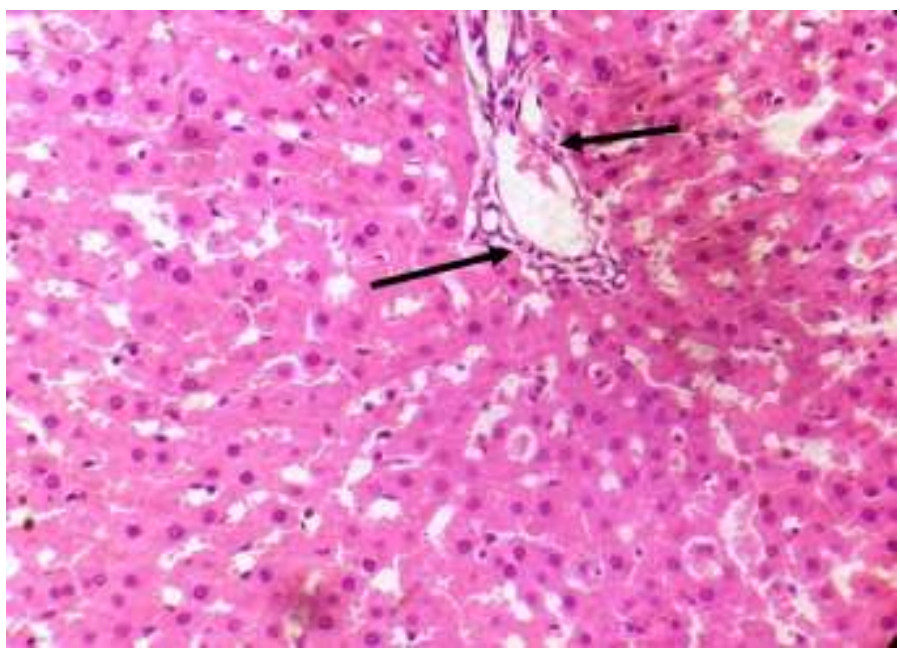

Plate 2. A piece of liver tissue of the experimental rats inoculated with $S$. aureus. Note the infiltration of inflammatory cells (arrows) around the pericentral area. H/E x200.

glomerular oedema (Plate 4) in high doses of the inoculum at the kidney.

The extent of cellular damage increased with more concentration of the inoculum. One remarkable feature of $S$. aureus is the tubular casts characterizing all values of the $S$. aureus inoculums in kidney tubules (Plate 5 ). The bladder upon inoculation with infective doses of 0.2 to 1.0 $\mathrm{ml} / \mathrm{kg}$ body weight of $\mathrm{S}$. aureus showed a mild effect on the musculature. The transitional epithelia of the bladder remained intact (Plate 6) with mild Infiltration of inflammatory cells which was in proportion of the infective doses of the inoculum.

In group II model of the experiment using graded doses

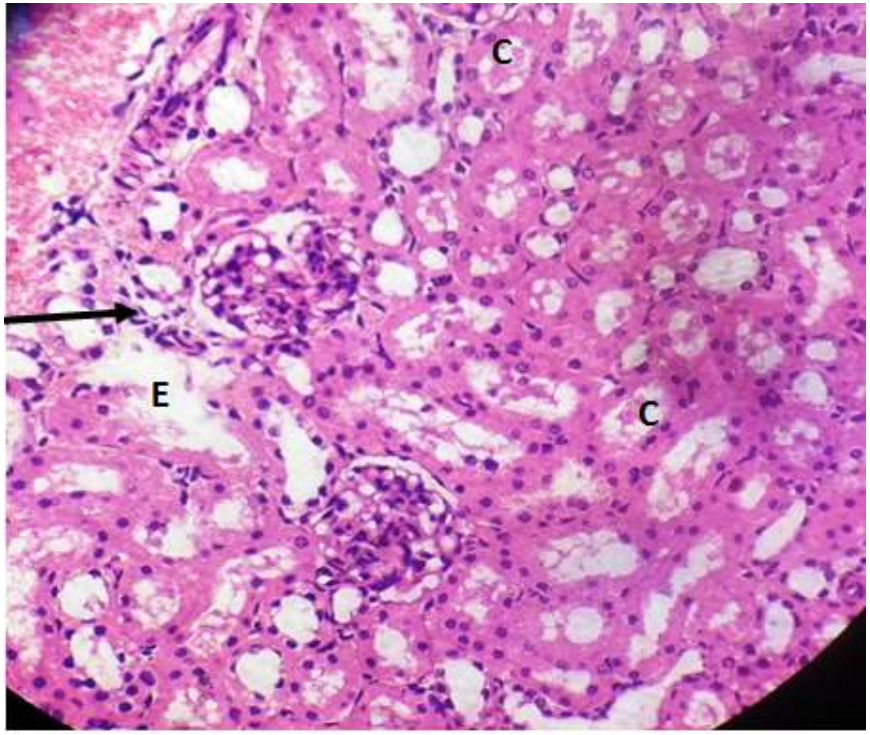

Plate 3. Kidney tissue from rat inoculated with $S$. aureus showing tubular casts (C) and tubular erosion (E) with inflammatory cells (arrow). H/E x200.

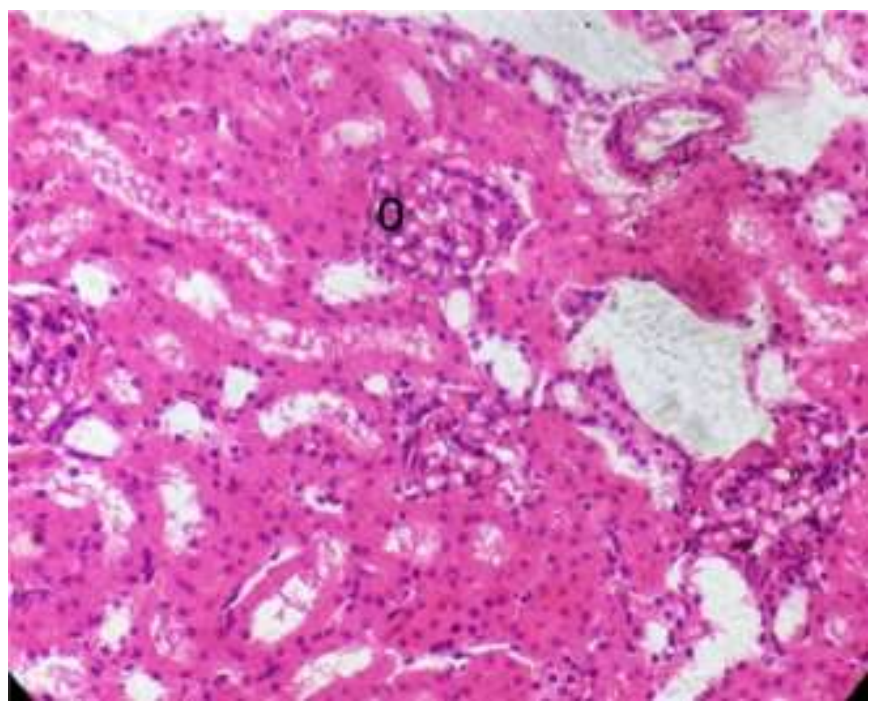

Plate 4. Demonstrates glomerular oedema (O) after $S$. aureus inoculation. $\mathrm{H} / \mathrm{E}$ x200.

of $S$. xylosus on the Wistar rats, the experiments $\mathrm{E}, \mathrm{F}$ and $G$ produced a relatively less degree of cell modification/alteration and damage. There was no visible alteration of the liver parenchyma. All hepatocytes of the rats' liver including those of the control rats were normal (Plate 7). There were tubular casts (Plate 8) in the kidney tubules. The glomerulus was mildly distorted with mild infiltration of inflammatory cells. Capsular oedema was noted in rats inoculated with high doses of $S$. xylosus. Tubular oedema was also seen in the stroma (Plate 9). 


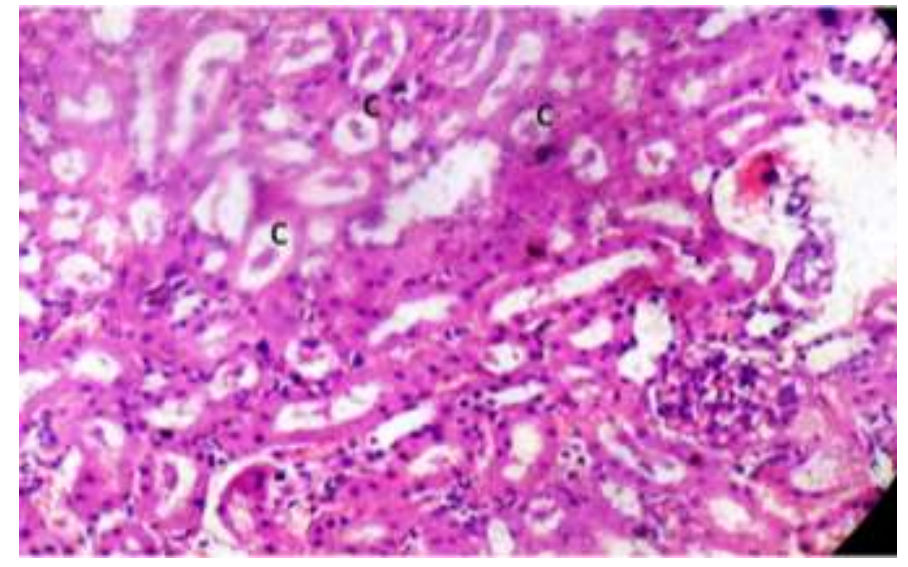

Plate 5. Kidney tubules in rats inoculated with $S$. aureus. Note the presence of tubular casts (C). H/E x200.

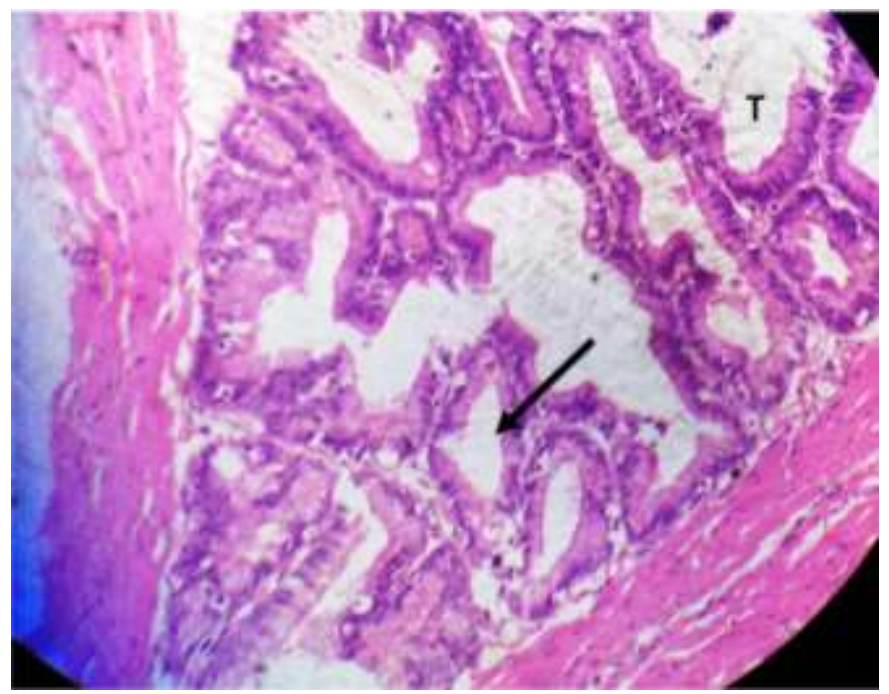

Plate 6. A piece of bladder tissue from rat following inoculation with $S$. aureus. The transitional epithelia $(\mathrm{T})$ were intact with mild infiltration of inflammatory cells (arrow). H/E x200.

In the bladder, the infective dose of $S$. xylosus inoculum did not affect the musculature of the bladder. There were inflammatory cells in the mucosae and connective tissues of the bladder (Plate 10). The transitory epithelia were intact and well defined (Plate 11). Group III model of experimentation using infective doses of $S$. lentus had a similar effect in the liver sections of rats inoculated. The liver showed no toxigenic effect. The central canals were intact, radiated round by the liver sinusoids. All doses $(0.2,0.5$ and 1.0$) \mathrm{mg} / \mathrm{kg}$ body weight of the infective doses of $S$. lentus was produced into glomerular oedema, inflammatory cells infiltration and tubular casts in proportion to the inoculated dose (Plate 12). The bladder did not manifest any major damage but showed mild epithelial erosion with inflammatory cells.

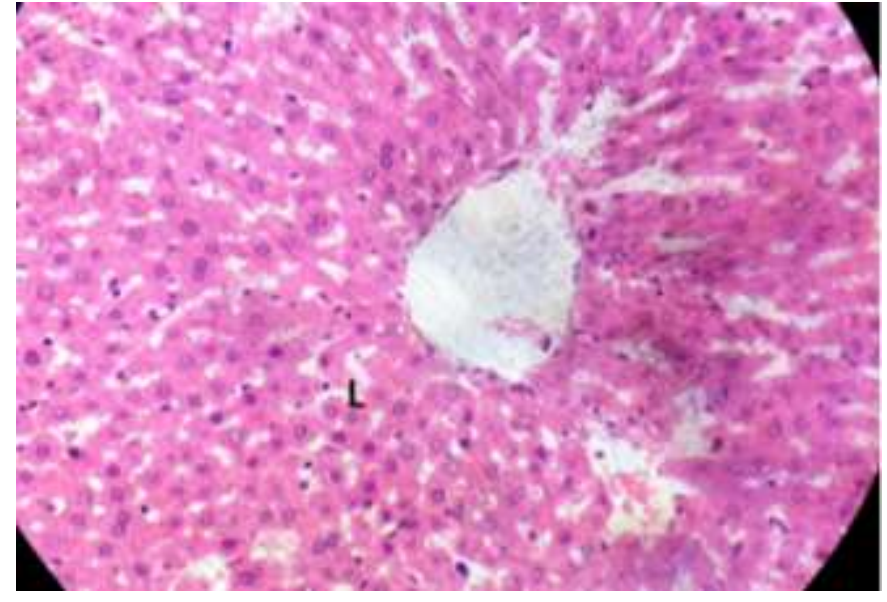

Plate 7. Showing intact liver parenchyma $(\mathrm{L})$ following inoculation with S. xylosus. H/E x200.

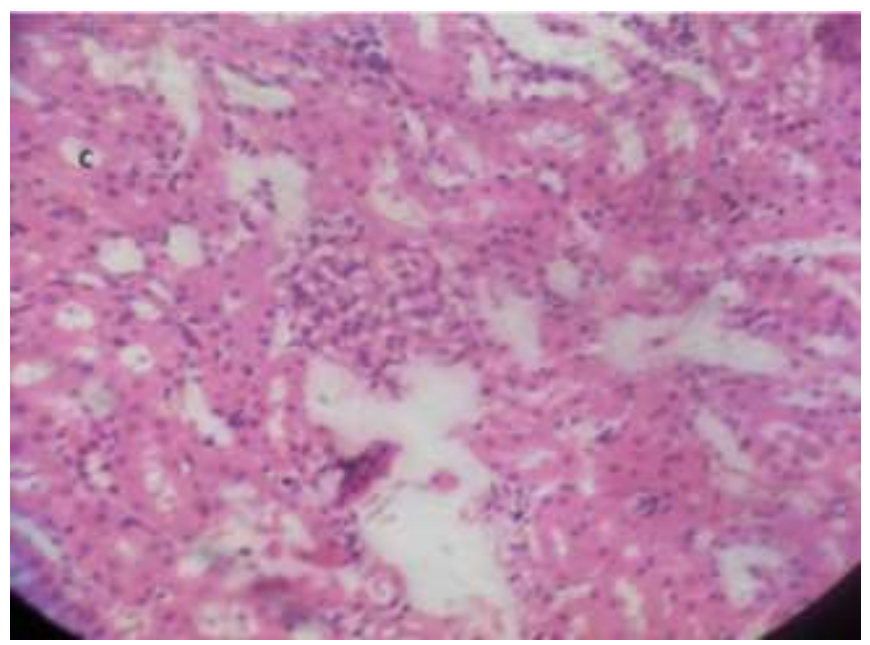

Plate 8. A piece of kidney tissue of rat inoculated with $S$. xylosus. Note the presence of tubular casts (C). H/E x200.

\section{DISCUSSION}

Mortality rate for the Wistar rats in the present study was $12.5 \%$ and occurred from intraperitoneal inoculation of the rats with S. xylosus 837 and $S$. aureus 856 strains. $S$. aureus produces enzymes such as coagulase, haemolysins, leucocidin, staphylokinase, lipase, hyaluronidase, protein $A$ and toxins such as enterotoxins and toxic shock syndrome toxin (Cheesbrough, 2010) which enables it to be invasive and pathogenic and these could be possessed by the $S$. aureus 856 test strains used in the present study which may have contributed to the 4 deaths recorded for it.

Moreover, $S$. aureus is a major cause of skin, soft tissue, bone, joint, lung, heart and kidney infections (Levinson, 2012), thus the rats may have suffered from generalized organ infections due to septicaemia 


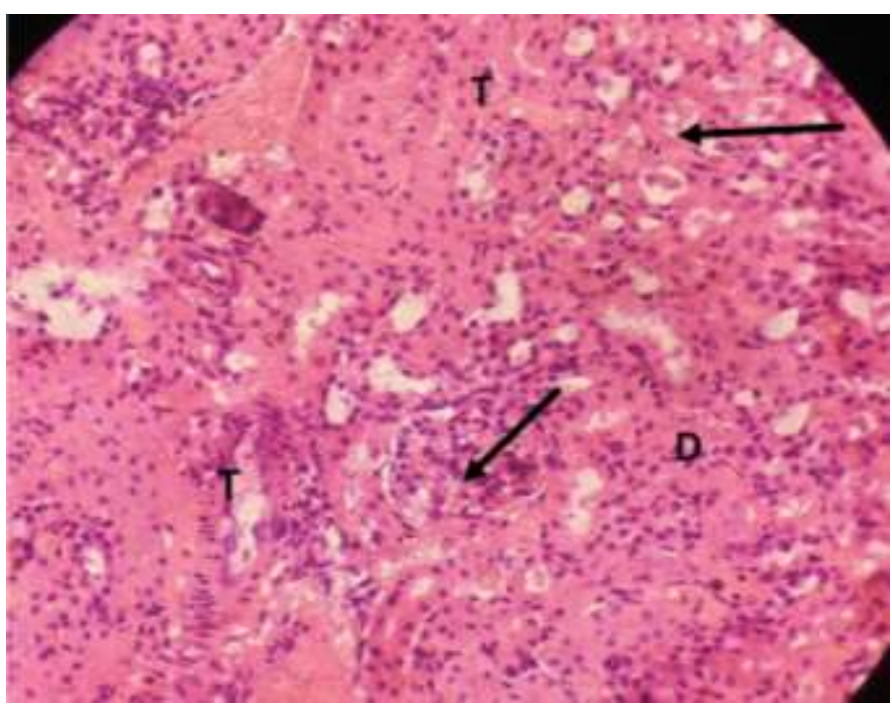

Plate 9. A piece of kidney tissue of rat inoculated with $S$. xylosus showing distortion of glomerulus (D); infiltration of inflammatory cells (arrows) and tubular oedema (T). H/E x200.

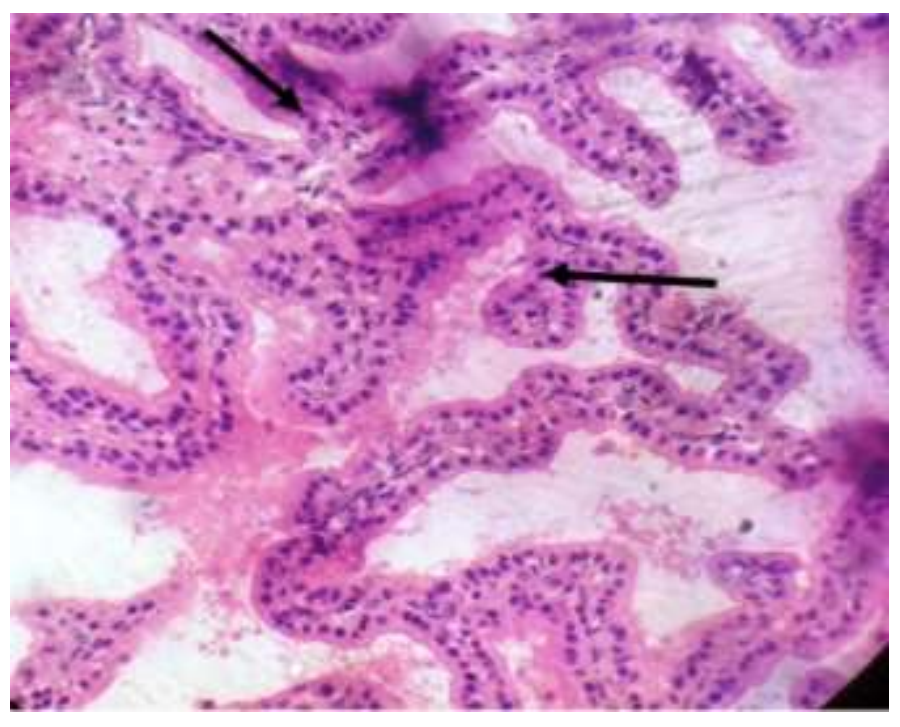

Plate 10. A piece of bladder tissue of rat showing inflammatory cells (arrows) in the mucosae and connective tissues following inoculation with $S$. xylosus. $\mathrm{H} / \mathrm{E} \times 200$.

leading to their deaths. The possession of virulent factors such as haemolysin, protease, lipase, and urease by $S$. xylosus could have contributed to deaths of the 8 rats inoculated with S. xylosus 837.

S. aureus 834 and S. lentus 853 did not cause deaths to any of the test animals in the present study, probably they are less virulent than the other Staphylococcus strains tested. Moreover, most staphylococci are said to be harmless and reside normally on the skin and mucous membranes of humans and other animals (Madigan and

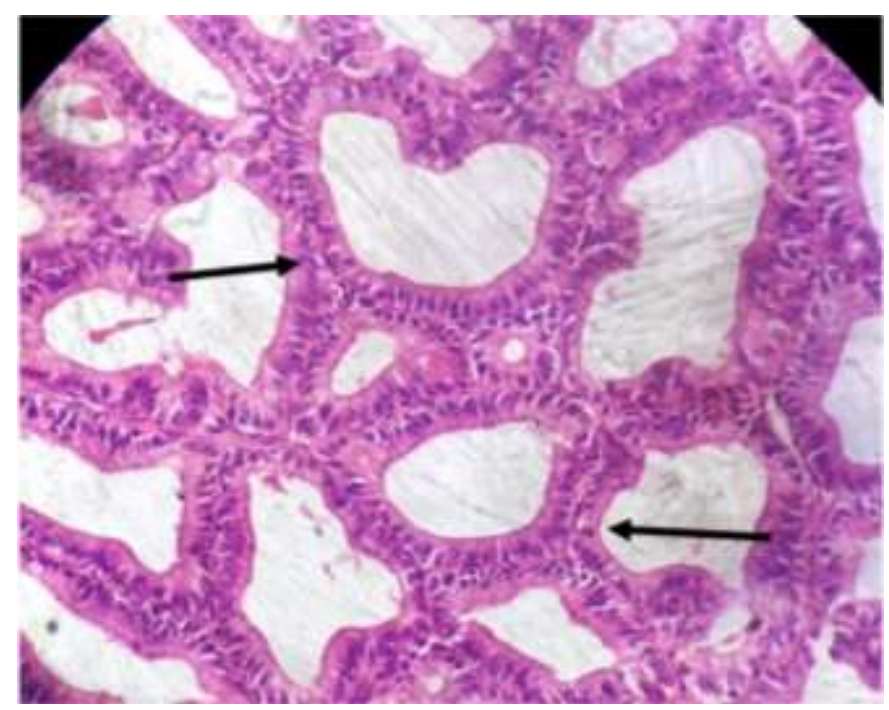

Plate 11. Bladder tissue showing normal epithelia (arrows) after inoculation with S. xylosus. H/E x200.

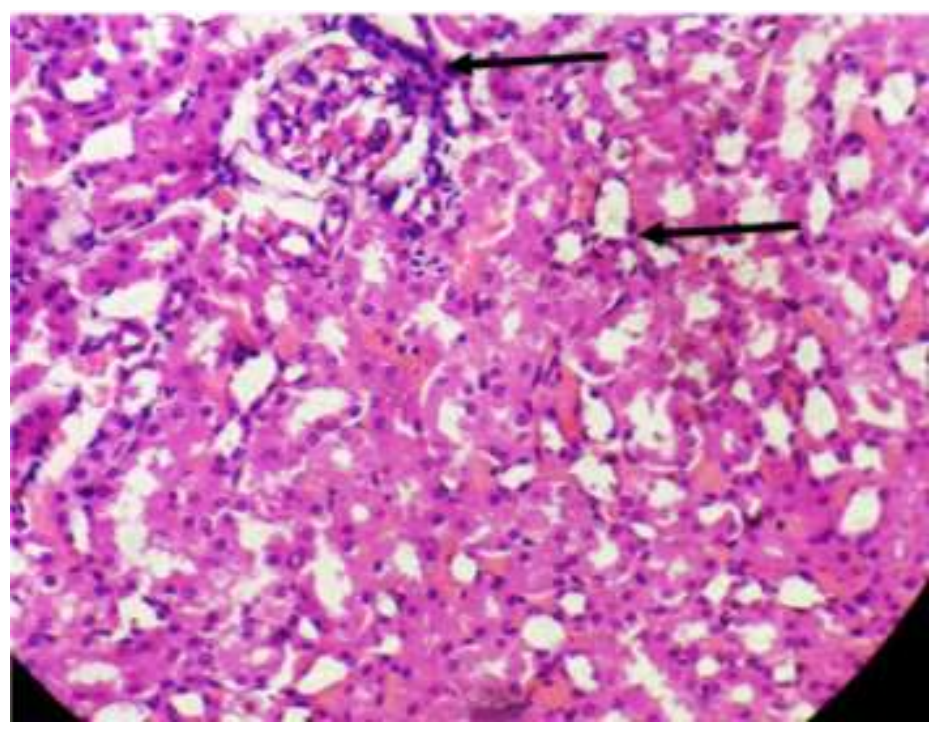

Plate 12. A piece of kidney tissue showing infiltration of inflammatory cells (arrows) after inoculation with $S$. lentus. $\mathrm{H} / \mathrm{E}$ x200.

Martinko, 2005) but could be opportunistic.

Of the 12 deaths that occurred in the present study, 8 $(66.7 \%)$ came from S. xylosus 837 in peptone water broth inoculation while $4(33.3 \%)$ was from $S$. aureus 856 peptone water broth inoculation, suggesting that $S$. xylosus 837 strain is more virulent than the $S$. aureus (856) strain. Of the eight deaths from S. xylosus 837 inoculation, males incurred more deaths $(6(75 \%))$ than females $(2(25 \%))$, so $S$. xylosus (837) strain seemed to be more virulent in male rats than in female rats. Of the 4 deaths from $S$. aureus (856) inoculation, all 4 deaths 
occurred in female rats (100\%) while none occurred in males $(0 \%)$ suggesting that $S$. aureus 856 may be more invasive in females than in males.

For $S$. xylosus 837 , mortality rate was equal at both 0.5 $\mathrm{ml}$ and at $1.0 \mathrm{ml}$ inoculations (four deaths occurring at each of the doses) representing $50 \%$ each and no death at $0.2 \mathrm{ml}$ inoculation. For $S$. aureus 856 , all the four deaths occurred at the highest dose of the inoculum 1.0 $\mathrm{ml}$ while none occurred at $0.2 \mathrm{ml}$ and at $0.5 \mathrm{ml}$ dose inoculations. The findings of the study suggest that mortality rates increased with increasing doses of the staphylococcal test strains.

Baba-Moussa et al. (2010) reported that $S$. aureus isolates from UTI in their work produced at least one enterotoxin. $21.5 \%$ of the $S$. aureus isolates from Cotonou University Hospital (CUH) in Benin used for their study produced Panton Valantine Leucocidine $(P V L)(P<$ 0.01). Six of 14 (43\%) PVL-positive isolates were methicillin resistant. Genes encoding clumping factor B, elastin and laminin binding proteins were detected in almost all isolates $(80 \%)$, hence they concluded that staphylococcal toxins and adhersion factors may be important in physiology of UTI. The finding of BabaMoussa et al. (2010) could have also contributed to pathological changes seen in the Wistar rats inoculated with graded doses of $S$. aureus 856 in the present study where the visceral organs of rats examined showed toxigenic modifications on the kidneys of the rats with presence of tubular casts, tubular erosions and glomerular oedema in high doses of the inoculum, and extent of cellular damage increased with more concentration of the inoculum, with tubular casts characterizing all concentrations of the $S$. aureus 856 inoculums in the kidney tubules. The bladder of the rats showed a mild effect on musculature. The transitory epithelia of the bladder remained intact with mild infiltration of inflammatory cells which was in proportion of the infective doses of the inoculum.

The work of Al-Mathkhury et al. (2008) on pathological study on $S$. xylosus isolated from patients with urinary tract infections showed that all strains of $S$. xylosus isolates from UTI in their study were able to produce protease, haemolysin while urease activity was variable. Pathological changes were caused by the $S$. xylosus Isolates on the mice used in their study which was represented by glomerulus shrinkage, haemorrhage, congestion and infiltration of inflammatory cells in the kidney while the urinary bladder suffered hydrophobic degeneration, dekeratinization as well as infiltration of inflammatory cells.

Similarities exists between the findings of Al-Mathkhury et al. (2008), and the result of the present study which shows that inoculation of the Wistar rats with graded doses of S. xylosus 837 produced tubular casts in the kidney tubules, mild distortion of the glomerulus with mild infiltration of inflammatory cells, capsular oedema in rats inoculated with high doses of S.xylosus 837, and tubular oedema seen in the stroma while the bladder of the rats showed inflammatory cells on the mucosae and connective tissues.

All doses $(0.2,0.5$ and 1.0$) \mathrm{ml} / \mathrm{kg}$ body weight of the infective doses of $S$. lentus produced glomerular oedema, inflammatory cells infiltration and tubular casts in proportion to the inoculated dose in the kidney while the bladder of rats did not manifest any major damage but showed mild epithelial erosion with inflammatory cells. Hence, S. lentus showed pathological potentials.

\section{Conclusion}

The present study has shown pathogenic and virulent potentials of two of the staphylococcal strains investigated S. xylosus 837 and S. aureus 856 with mortality rates for both at $12.5 \%$. S. xylosus 837 caused more deaths than $S$. aureus 856 suggesting higher virulent potential by $S$. xylosus 837 . S. xylosus 837 showed higher sex mortality rates in males than in females while $S$. aureus 856 demonstrated higher sex mortality in females than in males. Mortality rates increased in both staphylococcal strains with increased doses of their inoculums. The two test strains $S$. xylosus 837 and S. aureus 856 demonstrated several pathological changes on the kidney and bladder of Wistar rats and these changes heightened with increased doses of the test strains inoculum. The liver of the rats showed occasional mild infiltration of the inflammatory cells around the pericentral areas for $S$. aureus 856, no visible alterations of the liver parenchyma or hepatocytes by $S$. xylosus 837 and no toxigenic effect on the liver by $S$. lentus 853.

\section{CONFLICT OF INTERESTS}

The authors have not declared any conflict of interests.

\section{ACKNOWLEDGEMENTS}

The authors wish to thank the management of University of Nigeria Teaching Hospital (UNTH) Ituku-Ozalla and Enugu State University Teaching Hospital (ESUTH), Enugu, both in Enugu state, Nigeria, for granting ethical permission that enabled urine samples to be collected from their patients and other participants for this study. Thanks to all the participants who submitted their urine samples from which the staphylococcal isolates used for the study were obtained, and thanks to all who contributed in one way or the other to make this work a success.

\section{REFERENCES}

Alemu A, Moges F, Shiferaw Y, Tafess K, Kassu A, Anagaw B, Agegn A (2012). Bacterial profile and drug susceptibility pattern of urinary tract 
infection in pregnant women at university of Gondar Teaching Hospital North West Ethiopia. BMC Res Notes. 5:197.

Almathkuhury HJF, Flayyih MT, Abdullah AL-Ghrairy ZK (2008). Pathological study on Staphylococcus xylosus isolated from patients with urinary tract infections. J. Al-Nahrian University 11(2):123-130.

Amaeze NJ, Abah AU, Okoliegbe IN (2013). Prevalence and antibiotic susceptibility of uropathogens among patients attending University of Abuja. Int. J. Med. Med. Sci. 5(10):460-466.

Baba-Moussa L, Anani L, Scheftel JM, Couturier M, Reigel P, Haikou N, Hounsou F, Montel H, Sanni A , Prevost G (2008). Virulence factors produced by strains of staphylococcus aureus isolated from urinary tract infections. J. Hosp. infect. 68(1):32-38.

Bolaji RO, Onaolapo JA, Ibrahim YKE and Igwe JC (2013). Incidence of uropathogens from asymptomatic bacteriuric pregnant women in Zaria, Nigeria. Niger. J. Pharm. Sci. 12(1):1-8.

Brooks GF, Caroll KC, Butel JS, Morse SA, Mietzner TA (2013). Jawetz, Melnic and Adelbergs Medical Microbiology $26^{\text {th }}$ ed. Mc Graw-Hill companies Inc. USA. pp. 199-207.

Cheesbrough M (2010).Staphlocococcus aureus. District laboratory Practice in Tropical countries. Second edition update. Part 2 Cambridge low price ed. (Cambridge University Press South Africa). pp. 157-158.

Janakat S, Al-Merie H (2002). Optimazation of the dose and route of injection, and characterization of the time couse of carbon tetrachloride-induced hepatotoxicity in the rat. J. Pharmacol. Toxicol. Methods 48(1):41-44.

Levinson W (2012). Review of Medical Micobiology and Immunology, $12^{\text {th }}$ ed. MacGrawHill companies inc. USA. 710pp.

Madigan M, Martinko J (2005). Brock Biology of Microorganisms $\left(11^{\text {th }}\right.$ ed.) Prentice Hall ISBN 0-13-144329-1.
Mosbah H, Sayiri A, Bezzine S, Gargouri D (2006). Expression, purification and characterization of his tagged Staphylococcus xylosus lipase wild-type and its mutant Asp 290 Ala. Protein Express. Purif. 47(2):516-523.

Ochei J, Kolhatkar A (2000). Medical Laboratory Science Theory and Practice. Tata MacGraw- hill publishing company limited 7 West Patal Nagar New Delhi 110008, India. 1338p.

Oladeinde BH, Omoregie R, Olley M, Anunibe JA (2011). Urinary tract infection in a rural community of Nigeria. North Am. J. Med. Sci. 3(2):75-77.

Pragash DS, Giraja S, Shekar U, Rayupu V, Malathi (2014). Study of uropathogens among type II diabetic patients and their antimicrobial resistant pattern among rural south indian population. Sch. J. Appl. Med. Sci. 2(2B):589-591.

Wikipedia the free encyclopedia (2013). Staphylococcus xylosus http//en.wikipedia.org/w/index.php?title=Staphylococcus_xylosus\&old id $=542552341$ This page was last modified on 7 march 2013 at 09:34 and accessed on $14 / 6 / 2013$ 